\title{
Spectral functions at non-zero momentum in hot QCD
}

\section{Gert Aarts, Chris Allton, Justin Foley* and Simon Hands}

Department of Physics, Swansea University, Swansea, United Kingdom

E-mail: g.aarts@swansea.ac.uk, c.allton@swansea.ac.uk, j.foley@swansea.ac.uk, s.hands@swansea.ac.uk

\section{Seyong Kim}

Department of Physics, Sejong University, Seoul, Korea

E-mail: skimesejong.ac.kr

We present results for meson spectral functions at non-zero momentum at temperatures both below and above $T_{c}$, obtained in quenched simulations for a number of valence quark masses. For the lightest quark masses, a clear difference between the spectral functions on cold and hot lattices is observed.

XXIVth International Symposium on Lattice Field Theory

July 23-28, 2006

Tucson, Arizona, USA

${ }^{*}$ Speaker. 


\section{Introduction}

The experimental program at RHIC has generated much interest in the properties of the quarkgluon plasma (QGP) in the theoretical community, not least amongst lattice gauge theorists. Many of the dynamical properties of the QGP can only be determined by studying the relevant spectral functions $\rho(\omega, \mathbf{p})$. These are related to the corresponding euclidean-time correlators via an integral equation

$$
G(\tau, \mathbf{p})=\int_{0}^{\infty} \frac{d \omega}{2 \pi} K(\tau, \omega) \rho(\omega, \mathbf{p}), \quad K(\tau, \omega)=\frac{\cosh [\omega(\tau-1 / 2 T)]}{\sinh (\omega / 2 T)} .
$$

The survival of bound charmonium states in $N_{f}=2$ QCD well above the transition temperature [1], the calculation of dilepton and photon production rates [2] and the calculation of QGP transport coefficients $[3,4]$ are all topics which have been studied on the lattice. Determining a spectral function from a correlator, evaluated at a finite number of points in a lattice simulation, is an illposed problem. However, progress can be made by using the maximum entropy method (MEM) [5] to determine the most likely form for the spectral function given both the data and a set of prior assumptions for the spectral function. This prior information is encoded in a default model which is used in the MEM analysis, and one must be careful that the results obtained from this analysis are stable under reasonable variations of the default model.

We are particularly interested in calculating the hydrodynamical contribution in QCD spectral functions. Transport coefficients can be obtained from the slope of zero-momentum spectral functions at $\omega=0$, for example, the electrical conductivity is given by

$$
\sigma=\lim _{\omega \rightarrow 0} \frac{\rho_{e m}(\omega, \mathbf{0})}{6 \omega}
$$

where $\rho_{e m}$ is the spectral function for the conserved vector current. Unfortunately, lattice correlators are extremely insensitive to the details of spectral functions at small frequencies [6]. From general considerations, one knows that $\rho(\omega, \mathbf{p}) \sim \omega$ for $\omega \ll T$, and in the same regime the kernel can be written $K(\tau, \omega)=(2 T / \omega)+\mathscr{O}(\omega / T)$. Substituting these expressions into Eq. (1.1), one sees that the bulk of the contribution of the spectral function in the low frequency regime amounts to just a constant shift in the euclidean correlator. Therefore, one expects that the low-frequency part of a spectral function is the most difficult to compute using MEM.

As a first step, we have undertaken a study of meson spectral functions above the transition temperature. These functions are obtained from the euclidean correlators $G(\tau, \mathbf{x})=\left\langle J(\tau, \mathbf{x}) J^{\dagger}(0, \mathbf{0})\right\rangle$ where $J(\tau, \mathbf{x})$ are quark field bilinears

$$
J(\tau, \mathbf{x})=\bar{\psi}(\tau, \mathbf{x}) \Gamma \psi(\tau, \mathbf{x}), \quad \Gamma=1, \gamma_{5}, \gamma_{i} .
$$

We also computed these spectral functions for a number of values of spatial momentum. Changing the momentum allows us to investigate whether momentum-dependent features can be found, which may be helpful in identifying non-trivial hydrodynamic structure.

This work is particularly timely because there now exist predictions from AdS/CFT for finitemomentum spectral functions in $\mathscr{N}=4$ Super Yang-Mills theory [7, 8], and a comparison with lattice results is of immediate interest. 


\section{Simulation Details}

This study has been performed on sets of 100 quenched gauge configurations generated with a Wilson action on two lattices at temperatures both below and above $T_{c}$. The parameter values for these configurations are a subset of the values used in Ref. [9]. The 'cold' configurations were generated on a $48^{3} \times 24$ lattice with $\beta=6.5$. The temperature on this lattice was approximately $160 \mathrm{MeV}$. The second set of configurations were produced on a $64^{3} \times 24$ lattice with $\beta=7.192$. In this case $T \sim 420 \mathrm{MeV}$, which is about one and a half times the transition temperature. Staggered fermions were used for the valence quarks, and propagators were generated at three bare quark masses, $a m=0.01,0.05,0.125$. We are interested in comparing our results to hydrodynamic predictions and so we want to compute spectral functions for a number of momenta $p / T \lesssim 1$. However, for an isotropic lattice with periodic boundary conditions in the spatial directions, the lowest non-zero momentum in units of temperature is $p / T=2 \pi N_{\tau} / N_{\sigma}$, where $N_{\tau}$ is the number of lattice sites in the temporal direction and $N_{\sigma}$ is the extent in the spatial directions. On the hot lattice, this corresponds to a lowest non-zero momentum $p / T \sim 2.4$. To circumvent this problem and reach sufficiently small momenta, we use twisted boundary conditions for the valence quarks [10]. We computed propagators at four different twist angles and, using these together with Fourier transforms, we are able to access $\sim 20$ low-lying momenta.

\section{$2.1 Z_{3}$ symmetry}

It is well known that the pure gauge action has a global $Z_{3}$ symmetry. It is left unchanged under the multiplication of the temporal links by an element of $Z_{3}$, the centre group of $\mathrm{SU}(3)$. Of course, this is a symmetry of the quenched theory only and is broken by the fermion determinant appearing in the partition function of full QCD. The Polyakov line acts as an order parameter for the centre symmetry and the transition to the deconfined phase corresponds to spontaneous breaking of the $Z_{3}$ symmetry. The $Z_{3}$ vacua can be distinguished by the Polyakov loop expectation value, which can be real or complex: $\langle P\rangle=1, \exp ( \pm i 2 \pi / 3)$. In Ref. [11] it was shown for staggered fermions that the nature of the chiral transition in the real vacuum is markedly different from that of the vacua with complex Polyakov loops. Above $T_{c}$ in the real phase the chiral condensate drops to zero with vanishing quark mass, signalling the restoration of chiral symmetry. This is akin to what one might expect in full QCD. In the calculation of the quark propagators we chose our link variables such that the phase of the Polyakov loop is real, and observe a similar signal of chiral symmetry restoration: above $T_{c}$ for the lightest quark mass $m / T=0.24$ the pseudoscalar and scalar correlators are seen to coincide.

\subsection{MEM analysis}

For Kogut-Susskind fermions, the lattice correlator receives a contribution from an unwanted staggered partner. In this case the expression for the correlator in Eq. (1.1) becomes

$$
G(\tau, \mathbf{p})=2 \int_{0}^{\infty} \frac{d \omega}{2 \pi} K(\tau, \omega)\left[\rho(\omega, \mathbf{p})-(-1)^{\tau / a} \tilde{\rho}(\omega, \mathbf{p})\right]
$$

To compute $\rho(\omega, \mathbf{p})$, we perform independent MEM analyses on odd and even time slices and add the results to obtain the desired spectral function. In doing this, one has to be careful that the 

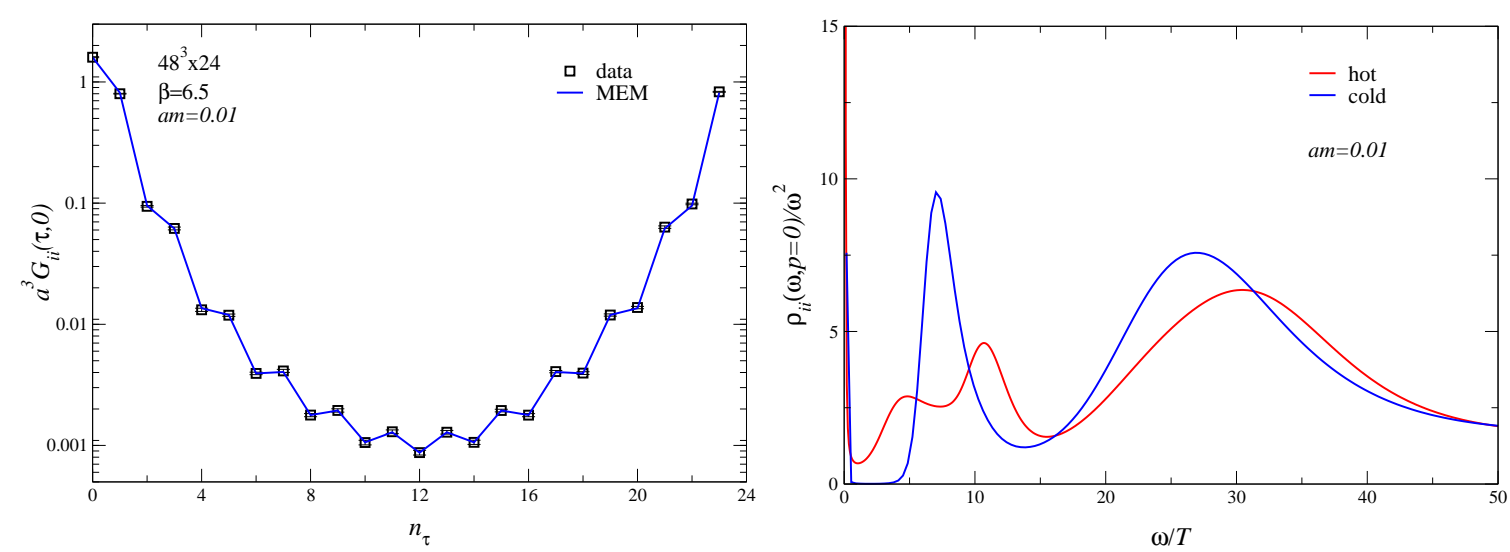

Figure 1: The plot on the left shows the reconstructed correlator in the vector channel plotted along with the original Monte Carlo data points. The right-hand side shows zero-momentum spectral functions in the vector channel both above and below $T_{c}$.

correlators defined on alternate time slices yield sensible, positive semi-definite spectral functions that can be handled by MEM. The main disadvantage of using staggered fermions compared to other fermion formalisms is that only half the available time slices are used in each MEM analysis. All the results presented here have been obtained using the standard Bryan analysis, although we have checked this approach against the classic and historic MEM methods. The default model initially used in our analysis is motivated by the expected behaviour of the spectral function in the continuum at asymptotically large frequencies $\rho_{\text {default }}(\omega) \sim \omega^{2}$.

\section{Results}

As a first check, we confirmed that it was possible to reconstruct the euclidean correlators from the results of the MEM analysis. The output for $\rho(\omega, \mathbf{p})$ and $\tilde{\rho}(\omega, \mathbf{p})$ were used to evaluate $G(\tau, \mathbf{p})$ by performing a simple numerical sum which approximated the integral in Eq. (2.1). An example of this is shown in the left-hand plot in Figure 1. This plots a reconstructed vector correlator, for the lightest quark mass, at vanishing spatial momentum below $T_{c}$, against the original numerical data points. The Monte Carlo results include a sum over the three vector polarisations. The agreement between the original data and the reconstructed data points is excellent, and we find this to be true in general provided that the data is of high quality with sufficiently small error bars. As one might expect, when the data is noisier the match between the reconstructed correlator and the original data is less precise.

In the confined phase below $T_{c}$, the spectral functions are peaked at energies which correspond to hadron resonances. Above $T_{c}$ the spectral functions look very different, particularly in the small frequency region. For example, the contribution from Landau damping means that these spectral functions are non-zero for all $\omega>0$. The right-hand side of Figure 1 shows vector meson spectral functions at zero spatial momentum above and below $T_{c}$. These have been rescaled by a factor of $\omega^{-2}$ to better reveal their detailed structure. In this plot, the melting of the hadronic state is evident. We also note that the spectral functions in both the hot and the cold phase show a large bump, centred at $\omega / T \sim 30$, which can attributed to lattice artifacts [12, 13]. 
Figure 2 shows the vector spectral function for varying values of spatial momentum. Below $T_{c}$ the position of the spectral function peak shifts to higher energies with increasing spatial momentum, and the corresponding dispersion relation yields a speed of light which is close to unity. Note also that, although we are in the cold phase, the temporal extent of the lattice $(\sim 1.2 \mathrm{fm})$ is not large enough to allow us to compute the bound-state energies using conventional lattice techniques, i.e. by fitting to the exponential decay of the correlator at sufficiently large times.

Above $T_{c}$, in the small $\omega$ region, there are very clear differences between spectral functions with different values of spatial momentum. In Figure 3, there appears to be a threshold energy which increases with increasing momentum. The spectral functions are odd functions of $\omega$ and the peak observed at $\omega \sim 0$ is an MEM artifact. Determining the precise origin of this artifact will require further analysis. For the purpose of this discussion, we adopt a naive approach and simply disregard the unphysical peak. The result of this is again shown in Figure 3. The spectral density has a bump at small values of $\omega$ which dies away with increasing momenta. This structure resembles the non-trivial structure expected from hydrodynamics and similar behaviour has been observed in an AdS/CFT calculation of spectral functions of the stress-energy tensor in $\mathscr{N}=4$ SYM [7].
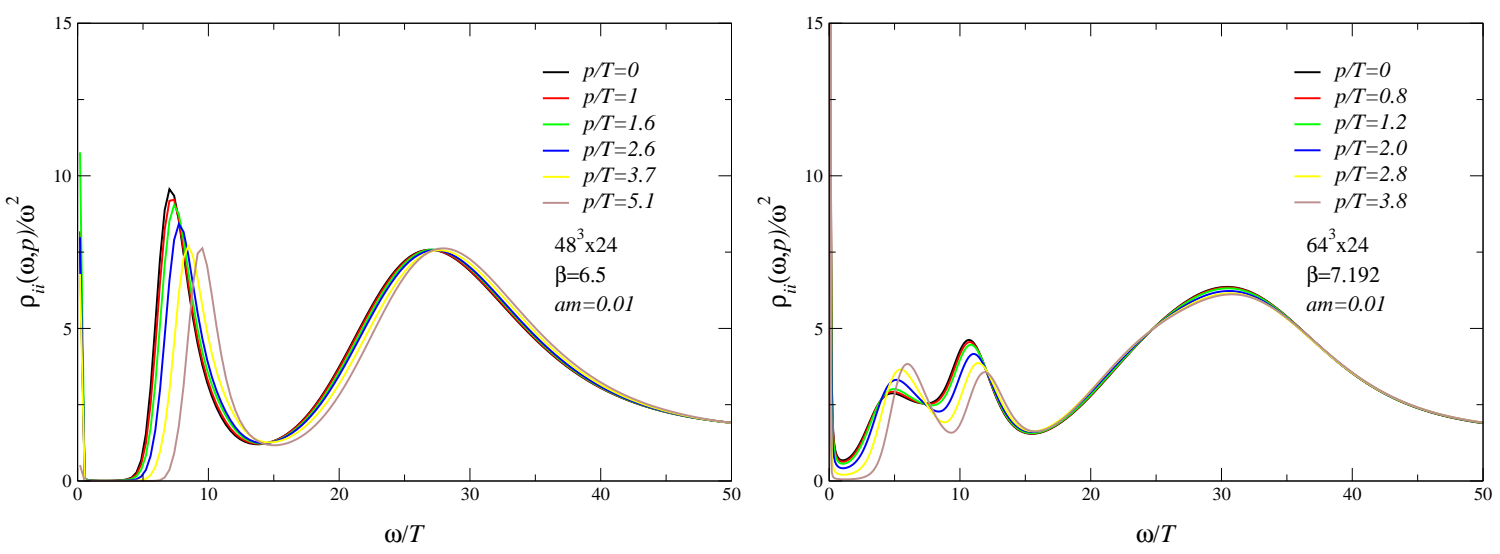

Figure 2: Vector spectral functions at the lightest quark mass for a range of momenta.

To test the reliability of these results, we repeated our analysis of the correlators with another default model. In this case we chose $\rho_{\text {default }}(\omega) \sim \tanh (\omega / \delta)\left(\delta^{2}+\omega^{2}\right)$ so that for $\omega \ll \delta$ the default model varies linearly with $\omega$ rather than quadratically, and it again scales as $\omega^{2}$ for $\omega \gg \delta$. For this analysis we took $\delta=0.1$. A comparison of the zero-momentum vector spectral functions obtained using the original and new default model is shown on the left-hand plot in Figure 4. Here, one can see that for $\omega / T \gtrsim 5$ the spectral functions are indistinguishable. Of course, there is no reason why they should differ, given that the two default models are virtually identical in this region. However in the small $\omega$ region considerable differences appear. A close-up of this is also shown in Figure 4. Once again, we have removed the unphysical spike at the origin. We note that at low frequencies the spectral functions obtained using different default models do not agree quantitatively, although this difference may be consistent with the uncertainty in the MEM 

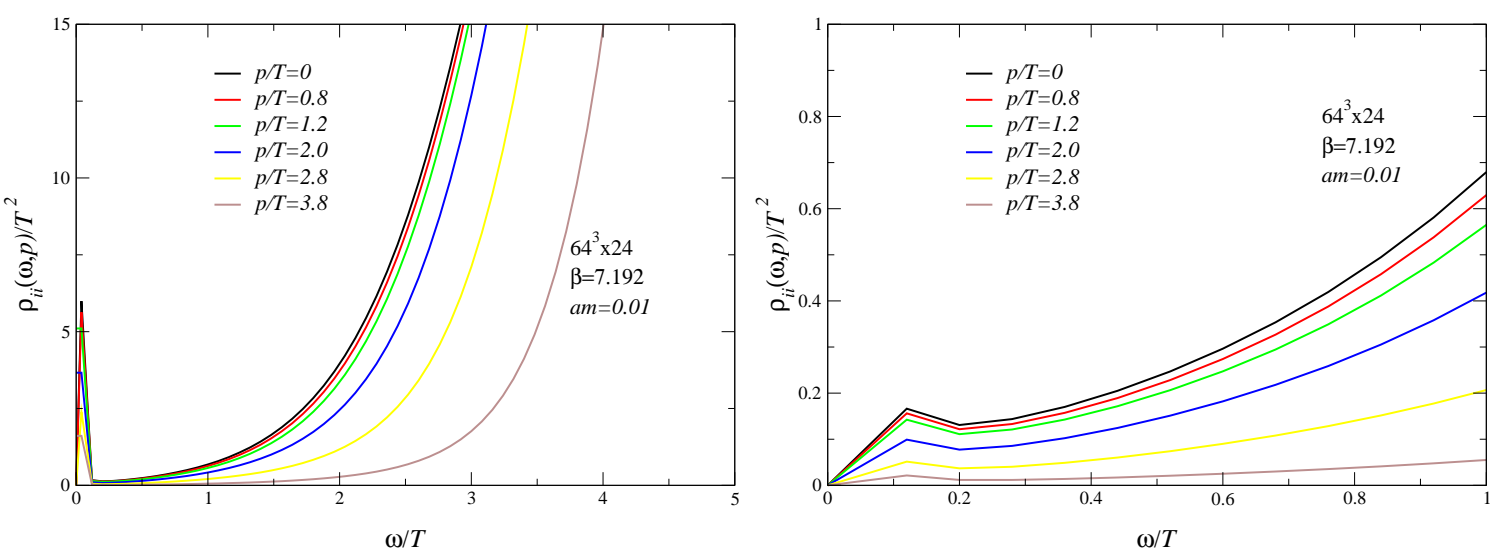

Figure 3: Close-up of the spectral functions in the hot phase shown both with and without the unphysical peak at $\omega=0$.
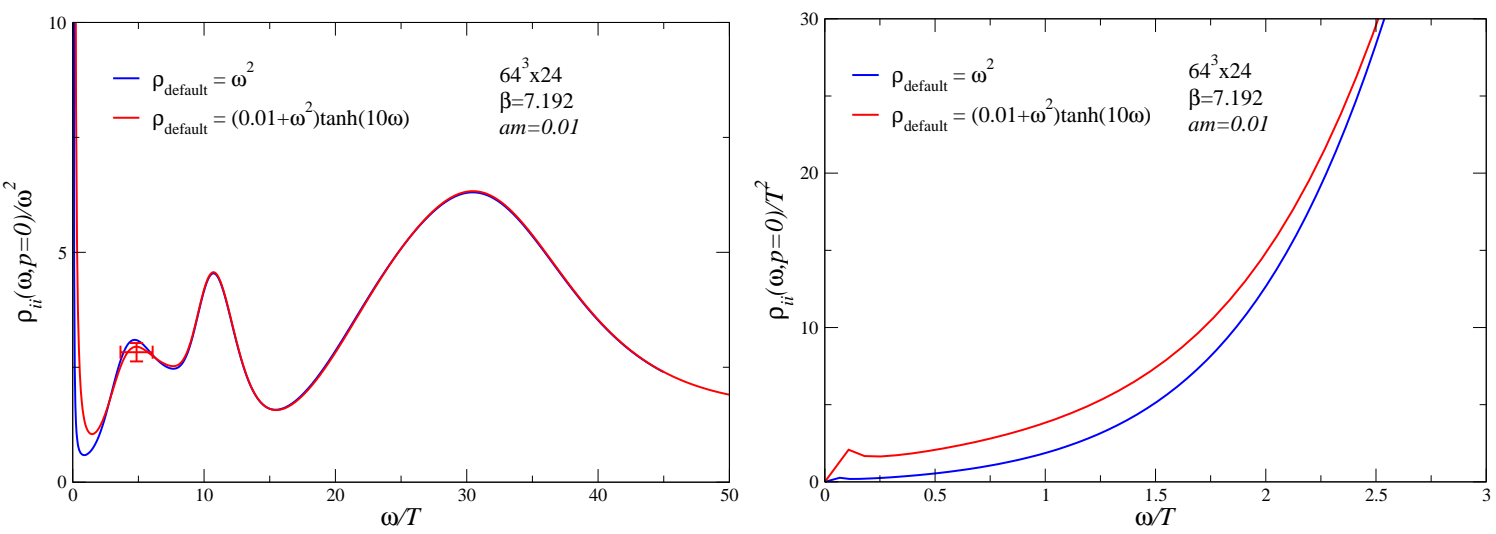

Figure 4: A comparison of zero-momentum vector spectral functions in the hot phase determined using different MEM default models as described in the text. The errorbar in the left-most plot gives an indication of the uncertainty in the MEM fi ts.

fit indicated by the errorbar in the left-hand figure. On the other hand, one might also argue that the original default model is completely at odds with the known behaviour of spectral functions for small $\omega$, and is not an appropriate default model for this regime. We also point out that, because of staggering in our correlators, we have used no more than twelve time slices in our analysis and it is possible that the results will converge as more time slices are used in the fit. Given the precise agreement between the reconstructed correlators and the Monte Carlo data shown earlier, this discrepancy highlights the fact that lattice correlators are remarkably insensitive to the details of the spectral function at low frequencies. This result indicates how difficult a precise determination of transport coefficients will be.

\section{Conclusions/Outlook}

We have computed meson spectral functions at non-zero momentum using the maximum entropy method. Preliminary results have been obtained both in the cold confined phase and in the hot phase above $T_{c}$. Twisted boundary conditions have been employed to access a range of low-lying 
momenta in order to look for non-trivial hydrodynamic structure. The maximum entropy method can clearly distinguish between spectral functions with differing momenta. Qualitatively, the spectral functions show little variation under a change of default model. For example in the confined phase the positions of peaks which identify hadronic bound-states appear to be stable. However, a quantitative determination of the spectral functions at low frequencies is still lacking, and further work is required.

The results presented here were obtained using staggered valence quark propagators. This is not the optimal choice for the MEM analysis. We are currently generating quark propagators using the non-perturbatively improved clover action [14]. This will allow us to include all time slices of the resulting correlators in the MEM analysis, which we hope will benefit the analysis.

Ultimately, it may be that a much more sophisticated approach, including, for example, the use of highly anisotropic lattices $[1,15]$, is required to precisely determine dynamical properties of QCD above $T_{c}$. However, the exploratory study described here certainly represents a progression towards this goal.

\section{References}

[1] G. Aarts, C. R. Allton, R. Morrin, A. P. O Cais, M. B. Oktay, M. J. Peardon and J. I. Skullerud, hep-lat/0608009; PoS LAT2005 176 (2006)

[2] F. Karsch, E. Laermann, P. Petreczky, S. Stickan and I. Wetzorke, Phys. Lett. B 530147 (2002)

[3] S. Gupta, Phys. Lett. B 59757 (2004)

[4] A. Nakamura and S. Sakai, Phys. Rev. Lett. 94072305 (2005)

[5] M. Asakawa, T. Hatsuda and Y. Nakahara, Prog. Part. Nucl. Phys. 46459 (2001)

[6] G. Aarts and J. M. Martinez Resco, JHEP 0204053 (2002)

[7] P. Kovtun and A. Starinets, Phys. Rev. Lett. 96131601 (2006)

[8] D. Teaney, Phys. Rev. D 74045025 (2006)

[9] S. Datta, F. Karsch, P. Petreczky and I. Wetzorke, Phys. Rev. D 69094507 (2004)

[10] J. M. Flynn, A. Jüttner and C. T. Sachrajda, Phys. Lett. B 632313 (2006)

[11] S. Chandrasekharan and N.H. Christ, Nucl. Phys. Proc. Suppl. 47527 (1996)

[12] F. Karsch, E. Laermann, P. Petreczky, S. Stickan, Phys. Rev. D 68014504 (2003)

[13] G. Aarts and J. M. Martinez Resco, Nucl. Phys. B 726 93-108 (2005)

[14] M. Lüscher, S. Sint, R. Sommer, P. Weisz and U. Wolff, Nucl. Phys. B 491323 (1997)

[15] R. Morrin, A. P. O Cais, M. J. Peardon and S. M. Ryan, Phys. Rev. D 74014505 (2006) 